\title{
FUNCTION GENERATOR BASED ON PERSONAL COMPUTER
}

\author{
Mohanad ABDULHAMID \\ AL-Hikma University, Iraq \\ moh1hamid@yahoo.com \\ Mutuku KAVITA \\ University of Nairobi, Kenya \\ researcher12018@yahoo.com
}

\begin{abstract}
This paper aims to generate the various waveforms commonly used in a laboratory. A computer is used to synthesize the waveforms. It makes use of software to synthesize the waveforms digitally and a Universal Serial Bus (USB) to parallel converter to transmit the digital version of the waveform to a digital to analog converter where it is converted to produce an analog waveform. A buffer is used to display the analog signal on an oscilloscope. Direct Digital Synthesis (DDS) is used to generate these waveforms. MATLAB simulation software is used to perform the $D D S$, and PROTEUS software is used for circuit design of the digital to analog converter.
\end{abstract}

KEYWORDS: function generator, personal computer, DDS, PROTEUS

\section{Introduction}

A function generator is a device, which generates various types of waveforms like the sine wave, the triangular wave, the square wave, the saw-tooth wave, the staircase wave, the cosine wave, the ramp wave etc. Normally, analog circuitry is employed for the generation and conditioning of waveforms. However the circuitry is usually complex, expensive and at times bulky. In this paper, a Personal Computer (PC) is used to emulate a function generator, through the use of software and hardware (Hui \& Ling, 2015; Zaheed, 2016).

A major advantage of a computer based function generator is that its output frequency, phase and amplitude can be precisely and rapidly manipulated under digital processor control. It has the advantage of fine frequency and phase resolution and the ability to rapidly hop between frequencies. In place of complex hardware, software is used. The digital circuits used to implement the function generator do not suffer the effects of thermal drift, aging and component variations associated with their analog counterparts. Another important advantage is that the use of software enables us to generate any type of waveform whose mathematical formula is known. This is not possible in the case of a normal function generator. Hence the output waveform of any signal whose trigonometric or algebraic formula can be obtained using a PC based function generator (Kokolanski \& Simic, 2017).

A function generator finds many applications in the laboratory. These include use in modulation, in clock generation, in telecommunications and in 
many other applications. There is therefore a need for a low cost, large bandwidth, versatile and simple function generator in the laboratory setting (Simic, Kokolanski, Denic, Dimcev, Zivanovic \& Taskovski, 2017).

\section{System Design}

2.1. Design of the Direct Digital Synthesis Computer Program

The flow chart shown in Figure no. 1 is used to write the DDS program in Matlab.

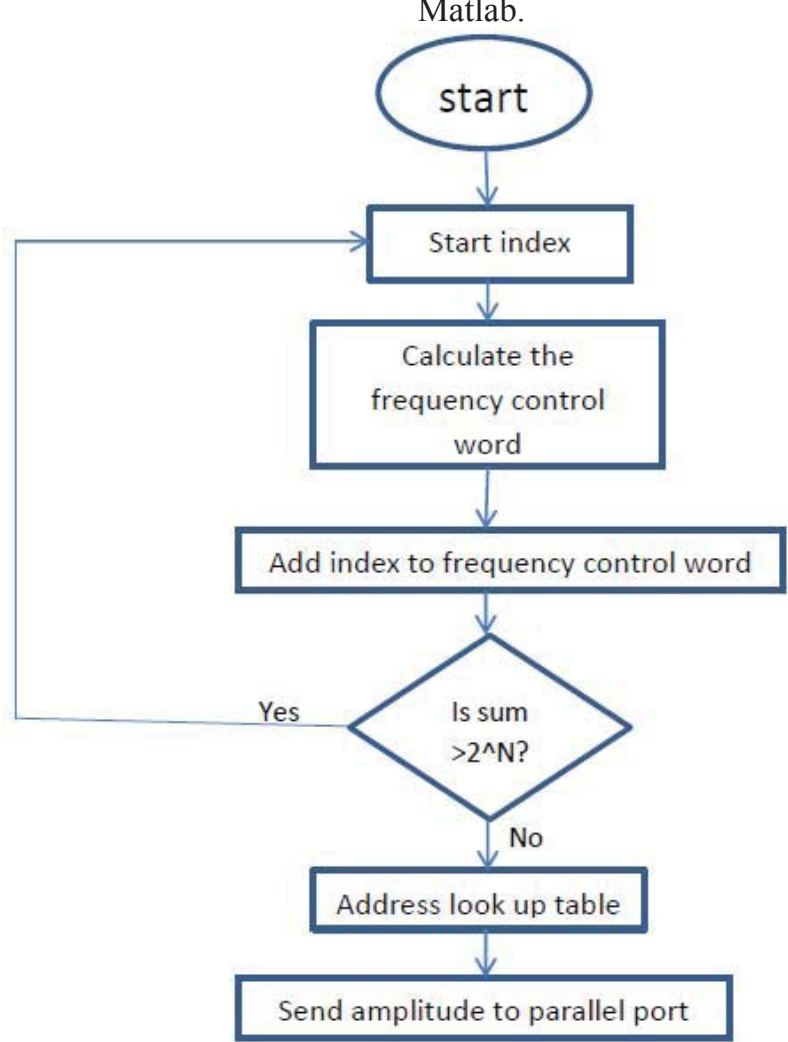

Figure no. 1: Flow chart for DDS
From the work specifications, the bandwidth required is from zero to $1 \mathrm{MHz}$. To satisfy Nyquist's criterion, to sample and reconstruct a $1 \mathrm{MHz}$ signal, then the minimum sampling frequency would have to be at least twice that of the signal being sampled. A sampling frequency of $4 \mathrm{MHz}$ is thus chosen.

For a maximum frequency of $1 \mathrm{MHz}$, a 20-bit accumulator is used. Both the frequency register and the phase register are set to be 20-bit. The size of the look up table is chosen to be eight bits since the output of the look up table is going to eight bits digital to analog converter and to minimize the effects of phase truncation.

\section{Converter}

\subsection{Design of the Digital to Analog}

There are three possible digital to analog converter configurations to choose from:

1) The weighted resistor digital to analog converter;

2) The R-2R ladder network;

3) A flash digital to analog converter (DAC). 
The R-2R ladder DAC is chosen because of the following reasons:

1) It requires only two different values of resistances;

2) It does not require precision resistors;

$3)$ It is cheaper to implement.

The R-2R ladder network would however perform conversion slower than the other two methods. An eight-bit R-2R ladder DAC is designed as shown in Figure no. 2. Because of the resistors in the DAC are expected to operate at a high frequency of $1 \mathrm{MHz}$, they are chosen to be of the metal film type. Carbon and metal wound resistors would be unable to operate at this frequency. The values of resistances used are $20 \mathrm{~K} \Omega$ and $10 \mathrm{~K} \Omega$ with a power rating of a quarter watt.

To buffer a $1 \mathrm{MHz}$ waveform, the minimum slew rate of summing amplifier is calculated to be $12.566 \mathrm{~V} / \mu \mathrm{S}$. For this slew rate and a gain bandwidth product of at least $1 \mathrm{MHz}$, the LM 318 operational amplifier is chosen since it has a slew rate of $50 \mathrm{~V} / \mu \mathrm{S}$ and a gain bandwidth product of $15 \mathrm{MHz}$. The summing amplifier is designed to operate as a single supply operational amplifier.

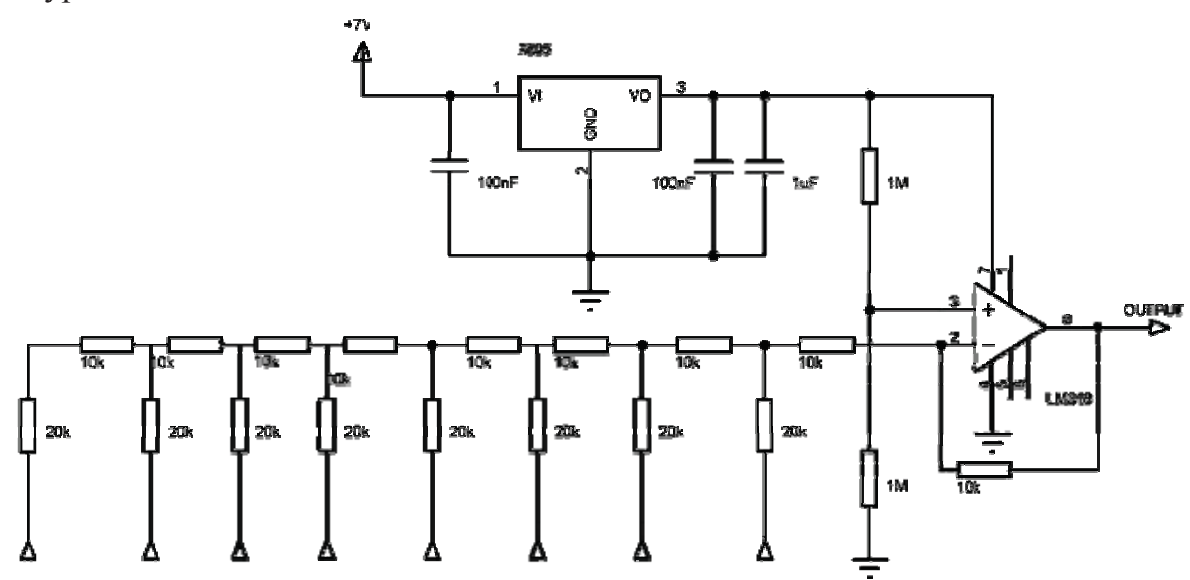

Figure no. 2: Digital to analog converter

\subsection{Design of the Computer} Interface

A high speed interface between the computer and the digital to analog converter is required. The interface is chosen to be an eight-bit USB to parallel converter. This is because of its very high data transfer rate. The parallel port can provide a maximum output current of $25 \mathrm{~mA}$. To protect the parallel port from damage, a buffer shown in Figure no. 3 is required to isolate it from the digital to analog converter. The buffer used is the 74HCT374 which is an octal D-flip flop. Its truth table is shown in Table no. 1, where:

$$
\mathrm{H}=\text { High Level (Steady State) }
$$

$$
\begin{aligned}
& \mathrm{L}=\text { Low Level (Steady State) } \\
& \mathrm{X}=\text { Don't Care } \\
& \uparrow=\text { Transition from Low to High } \\
& \mathrm{Qo}=\text { The level of } \mathrm{Q} \text { before the }
\end{aligned}
$$
Level indicated steady-state input conditions are established

$$
\mathrm{Z}=\text { High Impedance State }
$$

The bits on the input pins of the buffer appear on the output pins after each clock pulse. To clock the buffer, pin 14 of the parallel port is used. It is pulsed every time a data transfer between the parallel port and the digital to analog converter occurs. 


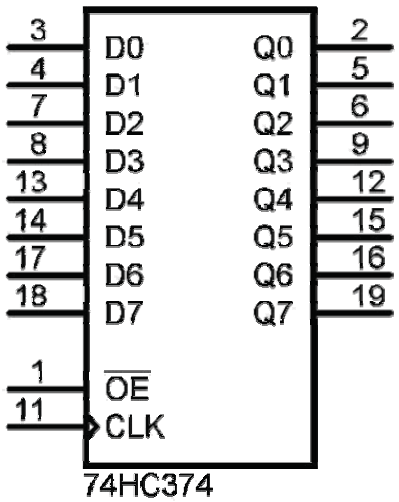

Figure no. 3: 74 HC374 buffer

Table no. 1

Truth table for 74 HC374 buffer

\begin{tabular}{|l|l|l|l|}
\hline \multicolumn{3}{|c|}{ INPUTS } & OUTPUT \\
\hline OE & CLK & Dn & Qn \\
\hline L & $\uparrow$ & H & H \\
\hline L & $\uparrow$ & L & L \\
\hline L & L & X & Qo \\
\hline H & X & X & Z \\
\hline
\end{tabular}

The pin out of the parallel port is shown Table no. 2 .

Table no. 2

Pin out for the parallel port

\begin{tabular}{|l|l|l|l|}
\hline DB-25 & SIGNAL NAME & DIRECTION & FUNCTION \\
\hline PIN & & & \\
\hline 1 & nStrobe & Out & Asserted by the computer to indicate the \\
\hline & & & presence of valid data on the data lines \\
\hline 2 & Data 0 & Out & Data line 0 \\
\hline 3 & Data 1 & Out & Data line 1 \\
\hline 4 & Data 2 & Out & Data line 2 \\
\hline 5 & Data 3 & Out & Data line 3 \\
\hline 6 & Data 4 & Out & Data line 4 \\
\hline 7 & Data 5 & Out & Data line 5 \\
\hline 8 & Data 6 & Out & Data line 6 \\
\hline 9 & Data 7 & Out & Data line 7 \\
\hline 10 & nAck & In & Negative pulse that indicates the reception \\
\hline & & & of the last character by the printer \\
\hline 11 & Busy & In & Asserted by the printer when it cannot \\
\hline & & & receive data (buffer is full, error) \\
\hline 12 & PError & In & Indicates end of paper \\
\hline
\end{tabular}




\begin{tabular}{|l|l|l|l|}
\hline DB-25 & SIGNAL NAME & DIRECTION & FUNCTION \\
\hline PIN & & & \\
\hline 13 & Select & In & Indicates online status of the printer \\
\hline 14 & nAutoFd & Out & Asserted by the computer to specify \\
\hline & & & automatic insertion by the printer of the \\
\hline & & & LF \\
\hline & & character after CR \\
\hline 15 & nFault & In & Indicates an error condition of the printer \\
\hline 16 & nInit & Out & Asserted to initialize the printer \\
\hline 17 & nSelectIn & Out & Asserted to select the printer \\
\hline 18 & Ground & & Ground for nStrobe signal \\
\hline 19 & Ground & & Ground for D0, D1 signals \\
\hline 20 & Ground & & Ground for D2, D3 signals \\
\hline 21 & Ground & & Ground for D4, D5 signals \\
\hline 22 & Ground & & Ground for D6, D7 signals \\
\hline 23 & Ground & & Ground for Busy, nFault signals \\
\hline 24 & Ground & & Ground for nAck, PError, Select signals \\
\hline 25 & Ground & & Ground for nAutoFd, nInit, nSelectIn \\
\hline & & & Signals \\
\hline
\end{tabular}

The complete circuit that is designed and implemented is shown Figure no. 4.

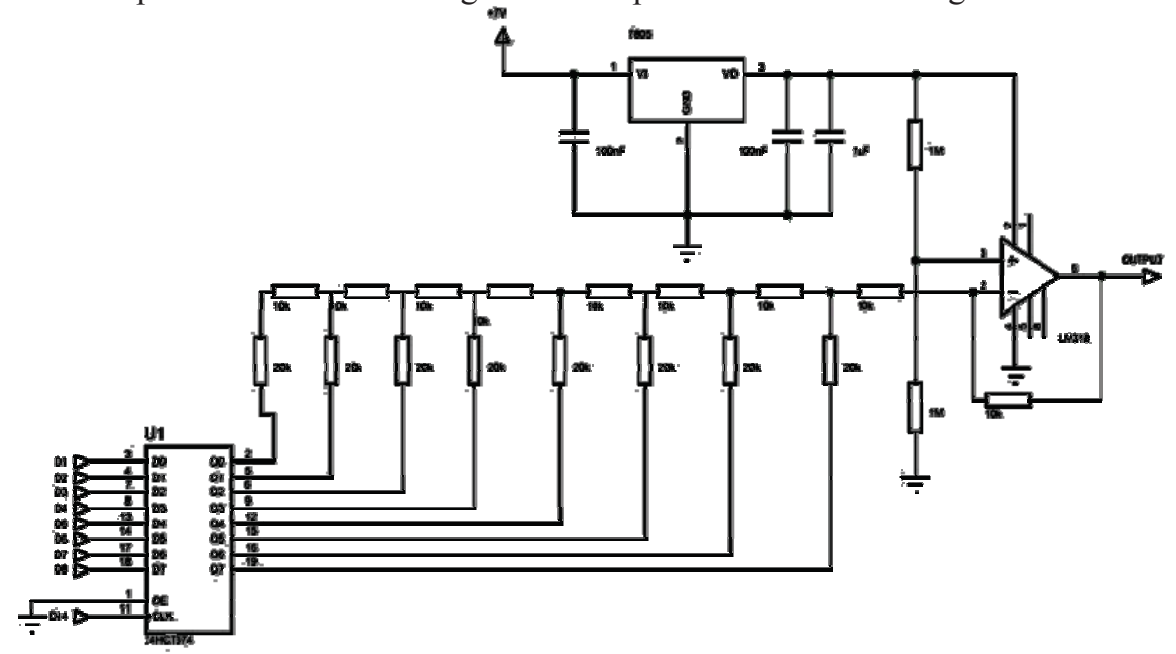

Figure no. 4: Complete circuit diagram

\section{Results}

\subsection{Simulation Results}

The results from the simulation of DDS in Matlab are obtained. The DAC being implemented is an 8-bit DAC, the look up table is set to be eight-bit long.
For the purposes of simulation, the program is written to generate only five periods of a sine wave. For an output frequency of $20000 \mathrm{~Hz}$, waveforms are obtained as shown in Figure no. 5, Figure no. 6, and Figure no. 7 . 


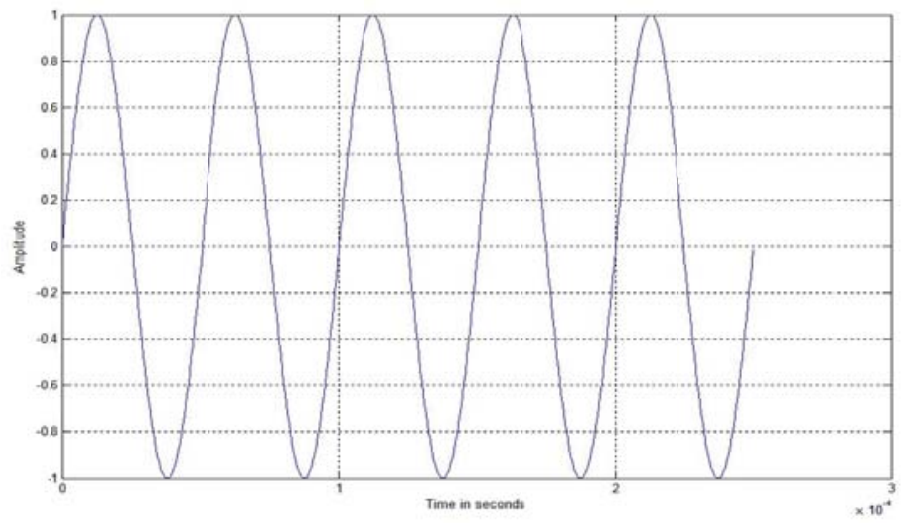

Figure no. 5: Simulated sine wave

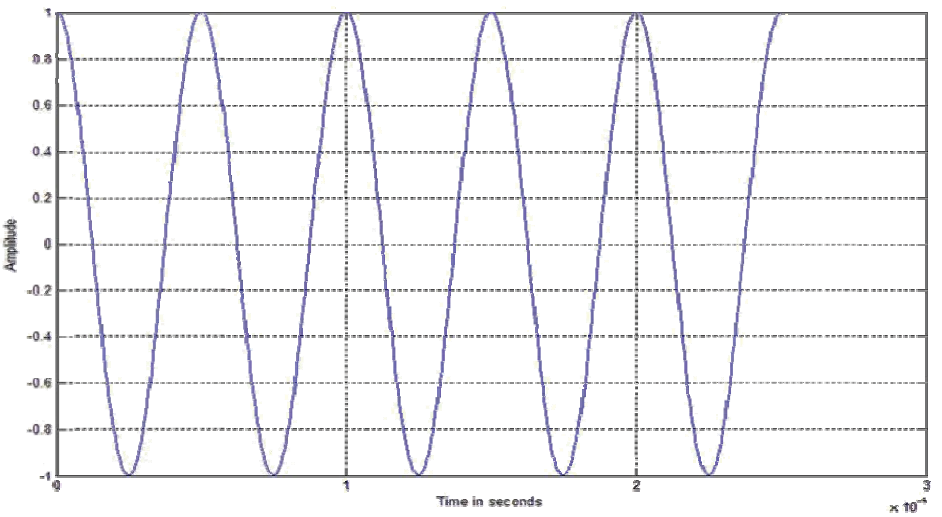

Figure no. 6: Simulated cosine wave

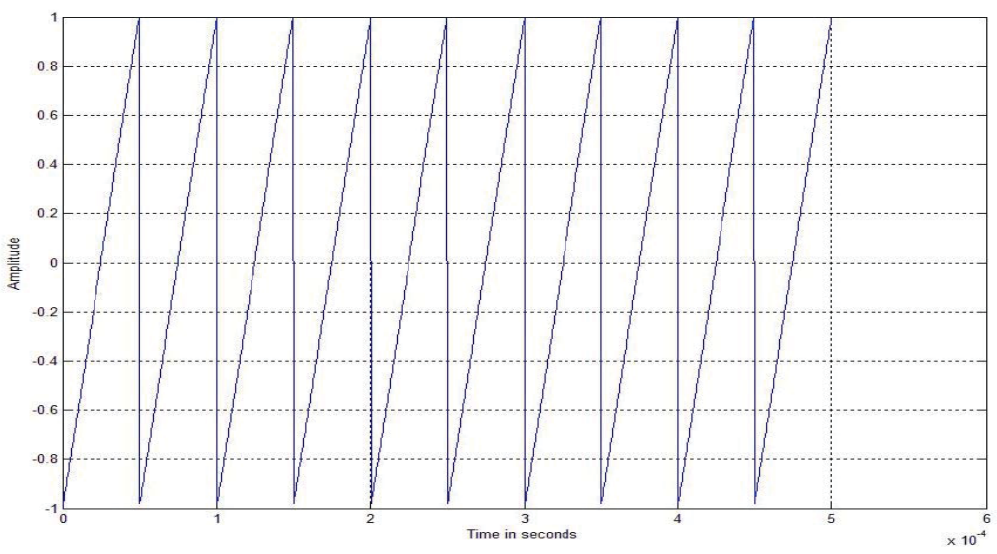

Figure no. 7: Simulated saw-tooth wave 


\subsection{Practical Results}

To determine whether the circuit would work practically, the generated samples are passed through the sound card of the computer. The sound card is a digital to analog converter with a bandwidth of $48000 \mathrm{~Hz}$. It can sample its input at a variable sampling frequency from zero to $48000 \mathrm{~Hz}$. For the experiment, a sampling frequency of $44000 \mathrm{~Hz}$ is chosen. This in effect set the frequency range of the sound card to $22000 \mathrm{~Hz}$. The sound card is very sensitive to loading. A buffer circuit using the LM 334 operational amplifier, shown in Figure no. 8 , is thus constructed to isolate the sound card from the oscilloscope. A monophonic audio jack is connected to the earphone port of the computer. Its ground is connected to the ground of the buffer circuit. Its other pin is connected to the non-inverting terminal of the operational amplifier. The buffer is designed to operate in its non-inverting mode in order to make use of its high input impedance for the isolation of the sound card.

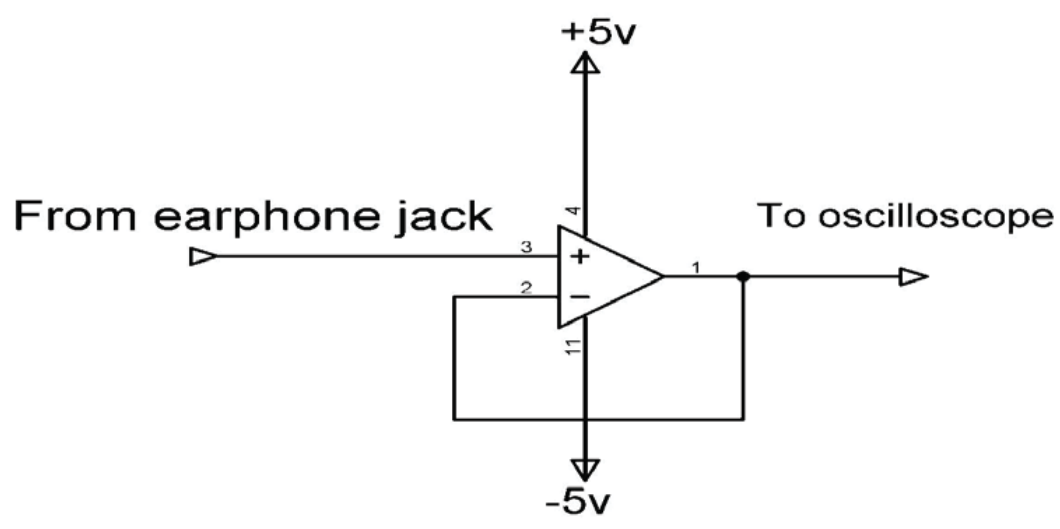

Figure no. 8: Buffer circuit

The waveform displayed on the oscilloscope is shown in Figure no. 9.

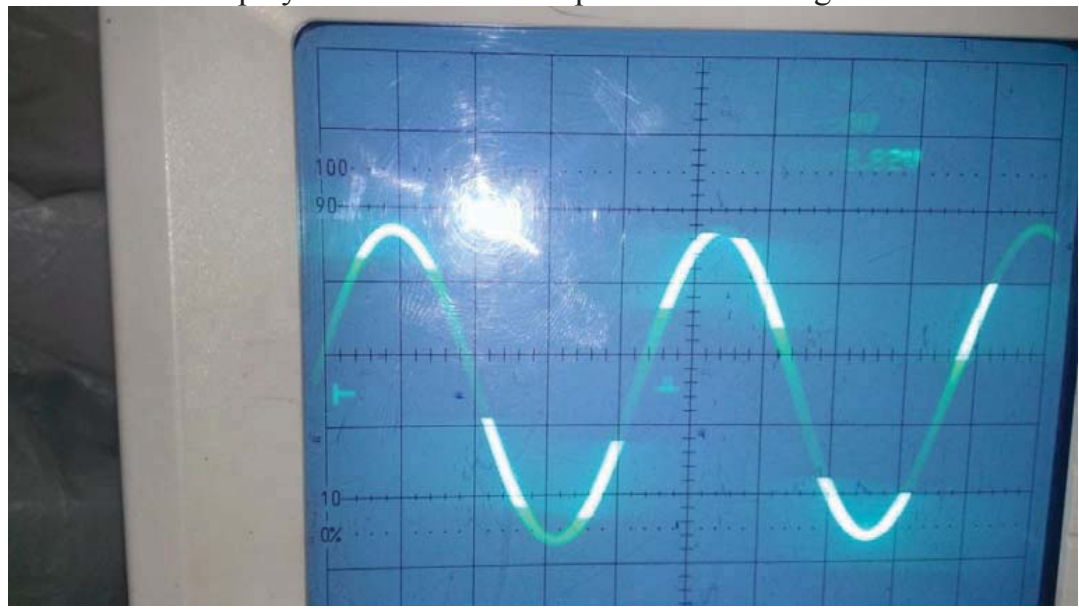

Figure no. 9: Practical result from sound card 


\section{Conclusion}

The work in this paper succeeded in synthesizing waveforms using software. It succeeded in creating an analog waveform using the sound card of the computer but failed to create an analog signal using the USB to parallel converter due to failure in the USB to parallel converter.

\section{REFERENCES}

Hui, Z., \& Ling, G. (2015). The design of signal generator based on virtual instrument. $3^{\text {rd }}$ Edition, International Conference on Machinery, Materials and Information Technology Applications, China.

Kokolanski, Z., \& Simic, M. (2017). Realization and testing of PC-based power quality signal generator. XXVI International Scientific Conference Electronics, Bulgaria.

Simic, M., Kokolanski, Z., Denic, D., Dimcev, V., Zivanovic, D., \& Taskovski, D. (2017). Design and evaluation of computer-based electrical power quality signal generator. Journal of Measurement, No. 107, 77-88.

Zaheed, S. (2016). Software based signal generator and oscilloscope. B.Sc. Project, BRAC University, Bangladesh. 